Electroacoustic absorbers: Bridging the gap between shunt loudspeakers and active sound absorption

Hervé Lissek, Romain Boulandet, Romain Fleury, and AJS

Citation: The Journal of the Acoustical Society of America 129, 2968 (2011); doi: 10.1121/1.3569707

View online: http://dx.doi.org/10.1121/1.3569707

View Table of Contents: http://asa.scitation.org/toc/jas/129/5

Published by the Acoustical Society of America 


\title{
Electroacoustic absorbers: Bridging the gap between shunt loudspeakers and active sound absorption
}

\author{
Hervé Lissek, ${ }^{a)}$ Romain Boulandet, and Romain Fleury \\ Laboratoire d'Electromagnétisme et d'Acoustique, Ecole Polytechnique Fédérale de Lausanne, Station 11, \\ CH-1015 Lausanne, Switzerland
}

(Received 10 June 2010; revised 18 February 2011; accepted 24 February 2011)

\begin{abstract}
The acoustic impedance at the diaphragm of an electroacoustic transducer can be varied using a range of basic electrical control strategies, amongst which are electrical shunt circuits. These passive shunt techniques are compared to active acoustic feedback techniques for controlling the acoustic impedance of an electroacoustic transducer. The formulation of feedback-based acoustic impedance control reveals formal analogies with shunt strategies, and highlights an original method for synthesizing electric networks ("shunts") with positive or negative components, bridging the gap between passive and active acoustic impedance control. This paper describes the theory unifying all these passive and active acoustic impedance control strategies, introducing the concept of electroacoustic absorbers. The equivalence between shunts and active control is first formalized through the introduction of a one-degree-of-freedom acoustic resonator accounting for both electric shunts and acoustic feedbacks. Conversely, electric networks mimicking the performances of active feedback techniques are introduced, identifying shunts with active impedance control. Simulated acoustic performances are presented, with an emphasis on formal analogies between the different control techniques. Examples of electric shunts are proposed for active sound absorption. Experimental assessments are then presented, and the paper concludes with a general discussion on the concept and potential improvements. (C) 2011 Acoustical Society of America.
\end{abstract} [DOI: $10.1121 / 1.3569707]$

PACS number(s): 43.38.Ew, 43.50.Ki, 43.20.El [AJS]

Pages: 2968-2978

\section{INTRODUCTION}

In the pioneering active noise control patent, ${ }^{1}$ the underlying principle lies in the processing of acoustic interferences, i.e. the cancellation of a primary sound wave with a controlled secondary source. This early formulation of active noise cancellation paved the way to a broad variety of active concepts, among which is active sound absorption. The formal introduction of the concept of sound absorption by electroacoustic means can be attributed to Olson and May, ${ }^{2}$ who applied a feedback control on a loudspeaker, based on sound pressure sensing. In the footsteps of this novel formulation of active noise control, Jessel et al. ${ }^{3}$ studied the principle of active absorbers in the light of formal analogies with the Huygens theory of sound propagation, leading to practical requirements of the secondary sound sources. Guicking ${ }^{4}$ extended the concept to an hybrid structure combining an acoustic passive absorber with an active electroacoustic transducer. This principle slightly evolved in the 1980s to turn into the concept of "smart foam." This concept aims at modifying the acoustic behavior of a polymer structure through a thin embedded polyvinylidene fluoride (PVDF) film and an acoustic feedback, where the whole acts both as a passive device and an active sound absorber.

At the same time, feedback techniques were developed introducing the concept of "direct impedance control" on a loudspeaker diaphragm. ${ }^{6}$ Here, the feedback employs a com-

\footnotetext{
a) Author to whom correspondence should be addressed. Electronic mail: herve.lissek@epfl.ch
}

bination of sound pressure and diaphragm velocity sensing, resulting in a broadband acoustic impedance control. ${ }^{7-9}$ In these feedback techniques, the collocation of the actuator and the sensor plays an important role in the stability and in the performances of the controlled device, especially in the cases where the active elements are distributed within arrays. ${ }^{10}$ This has inspired advanced control techniques, where the actuator is capable of self-sensing acoustic quantities. Indeed, the actuator is among one of the numerous components that rule the performances of active noise control, ${ }^{11}$ but the active noise control algorithms generally take little account of its dynamics and the means to modify its passive response to external sound pressure. Therefore it seems important to develop versatile techniques for better control of the loudspeaker dynamics that can be referred to as "actuator-based" active impedance control.

The first realizations of an actuator-based active feedback control can be found in the realm of audio engineering. ${ }^{12,13}$ In Ref. 13, an original velocity-feedback technique was employed with the objective of further extending the response of a loudspeaker in the low-frequency range. In this setup, the velocity information was processed through the differential voltage of a Wheatston bridge at the electrical terminals of the transducer, based on assumptions concerning the loudspeaker dynamics (i.e., motional feedback). This feedback resulted in a damping of the velocity response of the actuator around the resonance frequency, thus reducing the non-linear behavior in the low-frequency range. These actuator-based concepts also provide a smart and efficient solution for sensing acoustic quantities out of an electrical 
filter. For example, Lane et al. ${ }^{14}$ use a physical model of the electroacoustic loudspeaker within an active noise control device, with the objective of designing specific electrical filters dedicated to extract acoustic information out of electrical sensing.

In 1970 , Bobber ${ }^{15}$ showed that a transducer shunted by an electronic generator could be used for matching the transducer acoustic impedance to the sound field. Instead of targeting a local sound pressure reduction (as in Olson and May's paper $^{2}$ ), the active transducer is used to modify the acoustic particle velocity through a passive absorber to avoid any perturbation of the incident sound wave. When the conditions for impedance match are met, the electronic system operates as the characteristic impedance of an acoustic transmission line, thus serving as an absorber of sound energy. In the footsteps of this work, Elliott et al. ${ }^{16}$ suggested that a secondary sound source could be seen as an electrical network designed in order to match a specific load that maximizes the absorbed sound power. These observations paved the way to straightforward strategies for sound absorption through electroacoustic means, such as the concept of "shunt loudspeakers. ${ }^{17}$ It is proven that a simple electric resistance of positive value connected to the electric terminals of a loudspeaker can modify the value of the acoustic impedance of the diaphragm up to the point at which the loudspeaker system becomes an excellent absorber around its resonance frequency. Employing "negative" resistances (through negative impedance converters) further varies the acoustic impedance of the device. ${ }^{15}$ The performances of shunt loudspeakers can also be enhanced with an "hybrid feedback," 18,19 in which the acoustic impedance of a loudspeaker is broadly modified by connecting a negative resistance in series with a sound pressure-feedback. Since the negative impedance realizes motional feedback, ${ }^{13}$ the acoustic performances obtained with hybrid feedback are similar to the above-mentioned direct impedance control techniques.

All these passive and active control strategies have been extensively studied, but the equivalence between shunt and feedback control is not straightforward and is only suggested in a few papers. For example, Bobber ${ }^{15}$ presents electrical networks connected to a loudspeaker that allow the matching of the acoustic impedance of its diaphragm to the medium. However, a conceptual bridge between these different control techniques is still missing, which is one of the motivations of the present paper.

In the following, the "electroacoustic absorber" (EA) concept is introduced, inspired by Olson and May's and Bobber's articles, ${ }^{2,15}$ as well as by the techniques for substituting a negative resistance for a velocity-feedback presented by Lissek and Meynial. ${ }^{18,20}$ Section II introduces a strategy for synthesizing the acoustic impedance presented by a loudspeaker to the medium that can be performed by setting three independent parameters of a one-degree-of-freedom resonator. A synthetic formulation of the acoustic admittance resulting either from an electric shunt or an feedbacks on acoustic quantities is specifically developed. Then, acoustic feedbacks are shown to be equivalent to electric shunts that are formalized hereafter, allowing the synthesis of electric networks in view of active sound absorption. These develop-

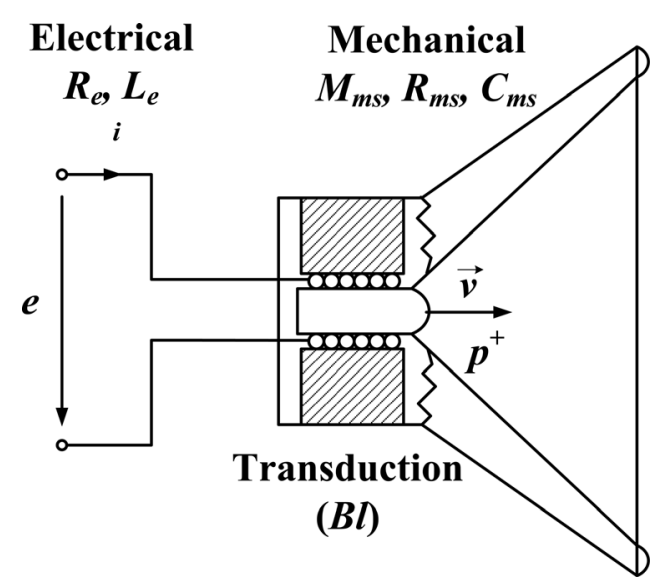

FIG. 1. Description of the electroacoustic loudspeaker and definition of parameters.

ments are followed by computational and experimental validations, as well as practical discussions on this unifying concept, with an emphasis on the analogies between the different control strategies.

\section{ELECTROACOUSTIC ABSORBER CONCEPT}

\section{A. General presentation}

In this paper, we consider a closed-box moving-coil loudspeaker radiating in a waveguide of adapted cross-section, where the assumption of plane waves under normal incidence is used throughout the formulations. Moreover, the following developments are restricted to the case of an electrodynamic transducer (see Fig. 1), but the presented results are also transposable to other transduction cases. ${ }^{15,21}$ The discussions focus on different feedback settings at the electric terminals of an electrodynamic transducer: A passive resistance, a velocity-feedback, and a direct active impedance control. The system as a whole (the electroacoustic transducer, the enclosure, and the electric feedback) is referred to as the electroacoustic absorber (EA).

A closed-box electrodynamic loudspeaker is a linear time-invariant system that, under certain hypotheses, can be described with differential equations. ${ }^{22}$ From Newton's law of motion, the mechanical dynamics of the loudspeaker diaphragm, for small displacements and below the first modal frequency of the diaphragm, can be modeled with the following linear differential equation:

$$
\begin{aligned}
S p^{+}(t)= & -M_{\mathrm{ms}} \dot{v}(t)-R_{\mathrm{ms}} v(t) \\
& -\left(\frac{1}{C_{\mathrm{ms}}}+\frac{\rho c^{2} S^{2}}{V_{b}}\right) \int v(t) \cdot d t-B l i(t),
\end{aligned}
$$

where $\rho$ is the density of the medium and $c$ is the celerity of sound in the medium, $M_{\mathrm{ms}}, R_{\mathrm{ms}}$, and $C_{\mathrm{ms}}$ are the mass, mechanical resistance, and compliance of the moving bodies of the loudspeaker, $V_{b}$ is the volume of the cabinet, $v(t)$ is the diaphragm velocity (opposed to total particle velocity), $B l$ is the force factor of the transducer (where $B$ is the magnetic field magnitude and $l$ is the length of the wire in the voice coil), $i(t)$ is the driving current, Bli(t) being the Laplace force 
induced by the current circulating through the coil, $S$ is the effective piston area, and $p^{+}$is the sound pressure at the outer (front) surface of the loudspeaker.

The electrical dynamics can also be modeled by a firstorder differential equation given as:

$$
e(t)=R_{e} i(t)+L_{e} \frac{d i(t)}{d t}-B l v(t),
$$

where $e(t)$ is the voltage applied at the electrical terminals, $R_{e}$ and $L_{e}$ are the dc resistance and the inductance of the voice coil, respectively, and $B l v(t)$ is the back electromotive force (EMF) induced by its motion within the magnetic field.

Equations (1) and (2) form a system of differential equations describing the loudspeakers dynamics. Expressing the preceding relationships with the use of Laplace transform yields the characteristic equations of the electrodynamic loudspeaker, given as:

$$
\left\{\begin{array}{l}
S P^{+}(s)=-\left(s M_{\mathrm{ms}}+R_{\mathrm{ms}}+\frac{1}{s C_{\mathrm{ms}}}\right) V(s)-B l I(s) \\
E(s)=\left(s L_{e}+R_{e}\right) I(s)-B l V(s)
\end{array},\right.
$$

where $P^{+}(s), V(s), E(\mathrm{~s})$, and $I(s)$ are the Laplace transforms of $p^{+}(t), \quad v(t), \quad e(t), \quad$ and $i(t), \quad$ respectively, and $1 /\left(C_{\mathrm{mc}}\right)=1 /\left(C_{\mathrm{ms}}\right)+\rho c^{2} S^{2} /\left(V_{b}\right)$ is the equivalent compliance due to the closed-box at the rear side of the loudspeaker.

The analytical formulation of the loudspeaker system can then be illustrated in the form of an equivalent circuit illustrated in Fig. 2, where $e_{s}$ and $R_{s}$ are the voltage source and its internal resistance, respectively. On the acoustic side, we assume an ideal exogenous sound source, $p_{S}$, located at one extremity of a waveguide facing the EA. The total acoustic pressure at the front side of the loudspeaker diaphragm, $p^{+}$, therefore corresponds to the addition of an incident sound pressure $p_{i}$ and a reflected sound pressure $p_{r}$, which accounts for the mechanical radiation impedance of the front face of the loudspeaker. However, this radiation impedance is excluded from the studied system with a view of providing general properties of the sound absorber (apart from the radiation conditions of the diaphragm). This impedance would not appear in the following developments.

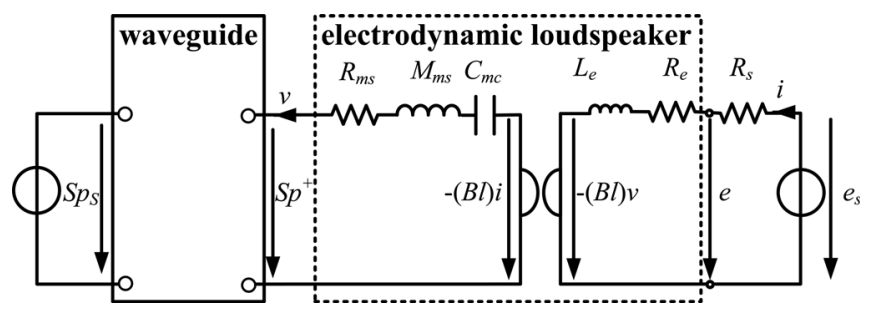

FIG. 2. Circuit representation of an electrodynamic loudspeaker including the electric load (electric mesh at the right of the loudspeaker). The acoustic disturbance is represented here by an ideal source of sound pressure $p_{S}$, and the waveguide is represented by a two-port system, accounting for the relationship between the input sound pressure $p_{S}$ and the sound pressure $p^{+}$at the front of the loudspeaker.

\section{B. Formulation of electroacoustic absorbers}

\section{Acoustic absorption capability of the speaker face}

It is always possible to derive the system of Eq. (3) in order to write the normalized acoustic admittance of the loudspeaker face as a function of the sound pressure $P^{+}(s)$ and velocity $V(s)$, whatever the load or feedback at its electrical terminals,

$$
Y(s)=-\rho c \cdot \frac{V(s)}{P^{+}(s)} .
$$

The minus sign is justified by the fact that $V(s)$ is defined as the diaphragm velocity, opposed to the total particle velocity at the diaphragm. The corresponding reflection coefficient can be derived after

$$
r(s)=\frac{1-Y(s)}{1+Y(s)}
$$

The extraction of the magnitude $|r(f)|$ of $r(s)$ yields the sound absorption coefficient $\alpha(f)$

$$
\alpha(f)=1-|r(f)|^{2}
$$

valid for the steady-state response of the system to harmonic excitations.

Equations (3)-(6) indicate that the choice of the electric load imposes certain absorption characteristics at the loudspeaker front face. The term "electroacoustic absorber" is thus justified when this load impedance $Z(s)$ is tailored in such a way as to exhibit positive values of the acoustic absorption coefficient.

This section aims at providing the general expression of the acoustic admittance presented at the loudspeaker diaphragm, when its electric terminals are connected to electric networks or acoustic feedback voltages. We consider here the voltage at the terminals of the loudspeaker as the combination of

(1) a feedback voltage on diaphragm velocity;

(2) a feedback voltage on sound pressure at the front face of the diaphragm;

(3) the source voltage lowering induced by the source electric resistance $R_{s}$.

The input voltage is therefore given by

$$
\begin{aligned}
E(s) & =E_{s}(s)-R_{s} I(s) \\
& =\Gamma_{v} V(s)+\Gamma_{p} P^{+}(s)-R_{s} I(s),
\end{aligned}
$$

where $\Gamma_{v}$ and $\Gamma_{p}$ represent the feedback gains, respectively, in $\mathrm{V} \mathrm{m}^{-1} \mathrm{~s}$ and $\mathrm{V} \mathrm{Pa}^{-1}$, including sensors sensitivities.

By replacing voltage $E(s)$ in Eq. (3) with the expression of Eq. (7) yields the normalized acoustic admittance:

$$
Y(s)=Z_{\mathrm{mc}} \frac{s^{2} a_{2}+s a_{1}}{s^{3} b_{3}+s^{2} b_{2}+s b_{1}+b_{0}},
$$


with

$$
\left\{\begin{array}{l}
a_{2}=L_{e} \\
a_{1}=R_{e}+R_{s}+\Gamma_{p} \frac{B l}{S} \\
b_{3}=L_{e} M_{\mathrm{ms}} \\
b_{2}=\left(R_{e}+R_{s}\right) M_{\mathrm{ms}}+L_{e} R_{\mathrm{ms}} \\
b_{1}=\left(R_{e}+R_{s}\right) R_{\mathrm{ms}}+\frac{L_{e}}{C_{\mathrm{ms}}}+B l\left(B l+\Gamma_{v}\right) \\
b_{0}=\frac{R_{e}+R_{s}}{C_{\mathrm{mc}}}
\end{array}\right.
$$

where $Z_{\mathrm{mc}}=\rho c S$ is the mechanical equivalent to characteristic medium impedance $Z_{c}=\rho c$. This expression can be simplified in the low-frequency range, below the cut-off frequencies $f_{e}$ and $f_{\text {me }}$ of the two electrical filters resulting from the connection of the electro-mechanical transducer to the electric load of Eq. (7) determined by,

$$
\left\{\begin{array}{l}
f<f_{e}=\frac{1}{2 \pi} \frac{R_{e}+R_{s}+\Gamma_{p} \frac{B l}{S}}{L_{e}} \\
f<f_{\mathrm{me}}=\frac{1}{2 \pi}\left(\frac{R_{e}+R_{s}}{L_{e}}+\frac{R_{\mathrm{ms}}}{M_{\mathrm{ms}}}\right)
\end{array}\right.
$$

and especially around the resonance frequency $f_{s}=1 /\left(2 \pi \sqrt{\left.M_{\mathrm{ms}} C_{\mathrm{mc}}\right)}\right.$. Without feedback, dc resistance $R_{e}$ generally being of the order of magnitude of $6 \Omega$ and inductance $L_{e}$ of about $1 \mathrm{mH}$, the above-mentioned cut-off frequencies are in the range of $1 \mathrm{kHz}$. The higher order terms in the numerator and denominator of Eq. (8) can be neglected, thus justifying the simplification of $Y(s)$ as

$$
Y(s) \approx Z_{\mathrm{mc}} \frac{s}{s^{2} M_{m E A}+s R_{m E A}+\frac{1}{C_{m E A}}},
$$

where

$$
\left\{\begin{array}{l}
M_{m E A}=M_{\mathrm{ms}} \frac{R_{e}+R_{s}}{R_{e}+R_{s}+\Gamma_{p} \frac{B l}{S}} \\
R_{m E A}=\frac{\left(R_{e}+R_{s}\right) R_{\mathrm{ms}}+\frac{L e}{C_{\mathrm{mc}}}+B l\left(B l+\Gamma_{v}\right)}{R_{e}+R_{s}+\Gamma_{p} \frac{B l}{S}} \\
C_{m E A}=C_{\mathrm{mc}}\left[1+\frac{\Gamma_{p} B l}{S\left(R_{e}+R_{s}\right)}\right]
\end{array}\right.
$$

are the mechanical equivalent components of the EA which exhibits the characteristics of a resonator. This set of parameters can also be replaced by the following set of parameters:

$$
\left\{\begin{array}{l}
f_{E A}=\frac{1}{2 \pi \sqrt{M_{m E A} C_{m E A}}} \\
\varsigma_{E A}=\frac{R_{m E A}}{Z_{\mathrm{mc}}} \\
Q_{E A}=\frac{1}{R_{m E A}} \sqrt{\frac{M_{m E A}}{C_{m E A}}}
\end{array}\right.
$$

$f_{E A}$ being the resonance frequency, $\zeta_{E A}$ being the normalized resistance, and $Q_{E A}$ being the resonance quality factor of the EA.
Thus, the EA concept allows easy determination of the parameters $R_{s}, \Gamma_{v}$, and $\Gamma_{p}$, in order to match the desired resonator parameters $\left(f_{E A}, \zeta_{E A}, Q_{E A}\right)$, opening the way to a straightforward control strategy for the active absorption of sound. A very singular result is the fact that the resonance frequency $f_{E A}$ is not affected much by the control, where any increase of pressure-feedback gain $\Gamma_{p}$ in positive values leads to the reduction of the apparent mass of the EA together with the increase of its compliance, thus a consecutive increase of the control bandwidth (inversely proportional to $Q_{E A}$ ). The sensitivity of the EA performances with the three control parameters is further detailed in Sec. III.

\section{Stability}

In order to anticipate stability issues, the Routh criterion is applied to the denominator of the expression of the normalized admittance, namely the coefficients $\left(b_{3}, b_{2}, \mathrm{~b}_{1}, b_{0}\right)$ of Eq. (8). By developing the Routh table, ${ }^{23}$ one can obtain the following parameters:

$$
\begin{aligned}
& s^{3} \\
& s^{2} \\
& s^{1} \\
& s^{0}
\end{aligned}\left(\begin{array}{ll}
b_{3} & b_{1} \\
b_{2} & b_{0} \\
c_{1} & c_{3} \\
c_{2} & c_{4}
\end{array}\right)
$$

where

$$
\left\{\begin{array}{rl}
c_{1}= & R_{\mathrm{ms}}\left(R_{e}+R_{s}\right)+\frac{L_{e}}{C_{\mathrm{mc}}}+B l\left(B l+\Gamma_{v}\right) \\
& -\frac{M_{\mathrm{ms}} L_{e}}{C_{\mathrm{mc}}\left[M_{\mathrm{ms}}+\frac{R_{\mathrm{ms}} L_{e}}{R_{e}+R_{s}}\right]} \\
c_{2}= & b_{0}=\frac{R_{e}+R_{s}}{C_{\mathrm{mc}}} \\
c_{3}= & c_{4}=0
\end{array} .\right.
$$

The condition for the stability yields each coefficient from the first column in the matrix of Eq. (14) should be of the same sign, yielding

$$
\left\{\begin{array}{l}
-R_{e}<R_{s} \\
\Gamma_{v}>\frac{1}{B l}\left[\frac{M_{\mathrm{ms}} L_{e}}{C_{\mathrm{mc}}\left[M_{\mathrm{ms}}+\frac{R_{\mathrm{ms} L e}}{R_{e}+R_{s}}\right]}-\frac{L_{e}}{C_{\mathrm{mc}}}-R_{\mathrm{ms}}\left(R_{e}+R_{s}\right)\right]-B l
\end{array}\right.
$$

One can then observe that if the first condition is satisfied, stability is always ensured if $\Gamma_{v} \geq 0$. This result is valid for the ideal linear model of Sec. II A, but it should be well understood that such a model does not account for various phenomena that may have a prejudicial impact on stability, such as the variation of electric resistivity and self-inductance with frequency, or non-linear behavior (stiffness of the suspensions induced by Laplace force), or even heat phenomena occurring in the coil. Such phenomena are not easy to model with accuracy but, in some cases, can be considered in a lumped-elements model. ${ }^{24,25}$ Nevertheless, the result of Eq. (16) is considered in the following developments, with a view of providing a criterion on stability for the ideal case. 


\section{Equivalent electric load}

It also follows from Eq. (3) and Eq. (7) that both velocity $V(s)$ and sound pressure $P^{+}(s)$ can be expressed as the functions of the electric current $I(s)$,

$$
E(s)=\Gamma_{v} V(s)+\Gamma_{p} P^{+}(s)-R_{s} I(s)=-Z(s) I(s),
$$

where $Z(s)$ represents the equivalent electric load impedance, ratio of the total control feedback voltage against current intensity. This electric impedance becomes:

$$
\begin{aligned}
Z(s)= & -\left(s L_{e}+R_{e}\right) \\
& -\frac{s^{2} L_{e}+s\left[\frac{\Gamma_{p} B l}{S}+R_{e}+R_{s}\right]}{s^{2} \frac{\Gamma_{p}}{S B l} M_{\mathrm{ms}}+s\left[\frac{\Gamma_{p}}{S B l} R_{\mathrm{ms}}-\left(1+\frac{\Gamma_{v}}{B l}\right)\right]+\frac{\Gamma_{p}}{S B l C_{\mathrm{mc}}}} .
\end{aligned}
$$

Each of the control cases described hereafter is then equivalent to an electrical network $Z(s)$, which is composed of a first negative series of resistance-inductance $-Z_{e}(s)=$ - $\left(s L_{e}+R_{e}\right)$, which can be viewed as a "neutralization" of the electric impedance of the loudspeaker, and a shunt impedance $Z_{s}(s)$ that depends on the control case. This neutralization reveals the required electric network that, connected to the loudspeaker, should fit the target acoustic admittance. In this sense, this formulation can directly be used for synthesizing electric networks capable of mimicking feedbackbased active absorption (e.g., Sec. III D). Conversely, each shunt has its acoustic feedback counterpart, namely a setting of the acoustic feedback gains $\Gamma_{p}$ and $\Gamma_{v}$ that plays the same role than the load impedance. Section III provides computational results, processed with the aforementioned formulations of the acoustic admittance and equivalent electric loads, for different examples of shunt and acoustic feedback controls that can be covered by the denomination "electroacoustic absorber."

\section{CASE STUDY}

In this section, the models are processed according to the following assumptions:

(1) An electrodynamic moving-coil loudspeaker (Visaton ${ }^{\circledR}$ AL 170 low-mid-range loudspeaker, the specifications of which are given in Table I) is used as the EA.

TABLE I. Electroacoustic transducer small signal parameters considered for the simulations.

\begin{tabular}{|c|c|c|c|c|c|c|}
\hline & \multicolumn{3}{|c|}{ Control settings } & \multicolumn{3}{|c|}{ Control results } \\
\hline & $\begin{array}{r}R_{s} \\
(\Omega)\end{array}$ & $\frac{\Gamma_{v}}{\left(\mathrm{~V} \mathrm{~m}^{-1} \mathrm{~s}\right)}$ & $\begin{array}{c}\Gamma_{p} \\
\left(\mathrm{~V} \mathrm{~Pa}^{-1}\right)\end{array}$ & $\begin{array}{c}f_{e a} \\
(\mathrm{~Hz})\end{array}$ & $\zeta_{e a}$ & $Q_{e a}$ \\
\hline Case 0 & $\mathrm{~N} / \mathrm{A}$ & $\mathrm{N} / \mathrm{A}$ & $\mathrm{N} / \mathrm{A}$ & 75.1 & 0.17 & 7.67 \\
\hline Case 1 & 5 & 0 & 0 & 74.9 & 1.05 & 1.25 \\
\hline Case $2 \mathrm{a}$ & 0 & 10 & 0 & 74.7 & 4.11 & 0.32 \\
\hline Case $2 b$ & 0 & 100 & 0 & 74.7 & 24.6 & 0.05 \\
\hline Case $2 \mathrm{c}$ & 0 & 194 & 0 & 74.7 & 443.3 & 0.003 \\
\hline Case $3 \mathrm{a}$ & 0 & 10 & 0.025 & 74.7 & 1.24 & 0.32 \\
\hline Case $3 b$ & 0 & 70.0 & 0.13 & 74.7 & 1.36 & 0.07 \\
\hline Case $3 c$ & 0 & 100 & 0.25 & 74.7 & 1.02 & 0.05 \\
\hline
\end{tabular}

\begin{tabular}{lccc}
\hline \hline Parameter & Notation & Value & Unit \\
\hline DC resistance & $R_{e}$ & 5.6 & $\Omega$ \\
Voice coil inductance & $L_{e}$ & 0.9 & $\mathrm{mH}$ \\
Force factor & $B l$ & 6.9 & $\mathrm{~N} \mathrm{~A}^{-1}$ \\
Moving mass & $M_{\mathrm{ms}}$ & 15.0 & $\mathrm{~g}$ \\
Mechanical resistance & $R_{\mathrm{ms}}$ & 0.92 & $\mathrm{~N} \mathrm{~m}^{-1} \mathrm{~s}$ \\
Mechanical compliance & $C_{\mathrm{ms}}$ & 1.2 & $\mathrm{~mm} \mathrm{~N}^{-1}$ \\
Effective area & $S$ & 133 & $\mathrm{~cm}^{2}$ \\
\hline \hline
\end{tabular}

TABLE II. Examples of setting cases and corresponding control results.

(2) The rear face of the loudspeaker diaphragm is enclosed in a box, the volume of which is $V_{b}=101$,

(3) The loudspeaker front face is radiating at the termination of a waveguide, the opposite extremity being considered as perfectly absorbent.

The different settings considered in this section are given in Table II. The acoustic absorption coefficients obtained by simulations are gathered on the synthetic illustration of Fig. 3, in order to show their common behavior and assess the influence of the EA parameters on the acoustic absorption coefficient on a single chart.

\section{A. Case 0: Open-circuit}

In the case where the electroacoustic transducer is not connected to any electric load, no current is circulating in the coil; thus no feedback force is created, and the device can be described as "passive." The acoustic admittance of the passive diaphragm can then be written as:

$$
Y_{0}(\mathrm{~s})=Z_{\mathrm{mc}} \frac{s}{s^{2} M_{\mathrm{ms}}+s R_{\mathrm{ms}}+\frac{1}{C_{\mathrm{mc}}}},
$$

This specific case provides insight on the behavior of other shunt and feedback techniques, since the admittance of

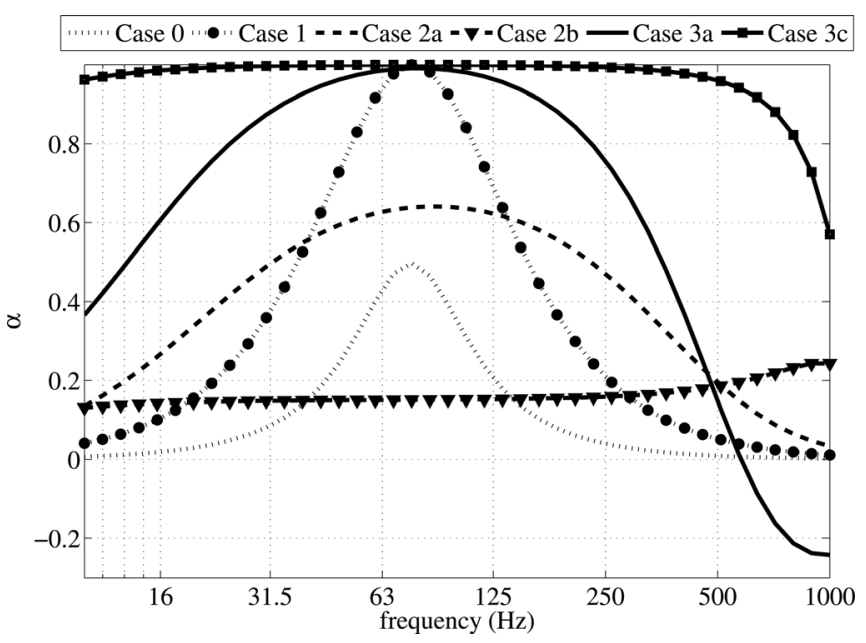

FIG. 3. Computed absorption coefficients of the EA for various setups (refer Table II). 
Eq. (11) is analog to Eq. (19). This expression exhibits a resonator behavior, the resonance frequency of which is determined by $f_{E A, 0}=f_{\mathrm{s}}=1 /(2 \pi) 1 / \sqrt{M_{\mathrm{ms}} C_{\mathrm{mc}}}$, the total losses being characterized by the normalized resistance $\zeta_{E A, 0}=R_{\mathrm{ms}} /\left(Z_{\mathrm{mc}}\right)$ and the resonance quality factor being $Q_{E A, 0}=1 /\left(R_{\mathrm{ms}}\right) \sqrt{M_{\mathrm{ms}} /\left(C_{\mathrm{mc}}\right)}$.

The acoustic absorption coefficient of the open-circuit EA is illustrated in Fig. 3, with the label "case 0," presenting a maximal value $\alpha_{0, \max }<1$ at the resonance. The "natural" resonant behavior of the passive loudspeaker is highlighted, with its relatively low capacity of absorbing the acoustic energy around its resonance frequency, due to mismatched mechanical losses (here $R_{\mathrm{ms}} \ll Z_{\mathrm{mc}}$ ). This also indicates that there is still some way to go before achieving total absorption at resonance, which has to be done by electrically adding losses in the system. This can be understood as an underlying objective of shunt techniques, as described in Sec. III B.

\section{B. Case 1: Shunt resistance (including shortcut)}

If we consider a single positive resistor $R_{s}$ loading the electric terminals of the loudspeaker, the normalized acoustic admittance can be expressed as follows:

$$
Y_{1}(s) \approx Z_{\mathrm{mc}} \frac{s}{s^{2} M_{\mathrm{ms}}+s\left(R_{\mathrm{ms}}+\frac{(B l)^{2}}{R_{e}+R_{s}}\right)+\frac{1}{C_{\mathrm{mc}}}} .
$$

In this expression, the mechanical resistance of the transducer can be increased by $(B l)^{2} /\left(R_{e}+R_{s}\right)$ expressing additional losses in the electric circuit. As a consequence, the absorption coefficient at the resonance can be easily varied, so as to cater for different values from $\alpha_{0, \max }$ up to 1 . This can also be explained by the fact that the positive electric resistance, fed by the induced back electromotive force, creates an electrical current in the coil. This current generates a feedback force at the loudspeaker diaphragm, modifying its vibrating velocity in response to an exogenous sound pressure. Thus, the loudspeaker connected to a passive shunt can no longer be denoted as "passive," as in the case of the open-circuit, and rather calls for the label "semi-active," due to this intrinsic feedback force.

An optimal shunt value can be set so as to have a perfect acoustic absorption at the transducer resonance,

$$
R_{\mathrm{opt}}=\frac{(B l)^{2}}{Z_{\mathrm{mc}}-R_{\mathrm{ms}}}-R_{e} .
$$

For the case of the studied electrodynamic loudspeaker in the air at $20{ }^{\circ} \mathrm{C}\left(\rho=1.204 \mathrm{~kg} \mathrm{~m}^{-3}\right.$ and $\left.c=343.3 \mathrm{~m} \mathrm{~s}^{-1}\right)$, this optimal resistance $R_{\mathrm{opt}}$ is nearly equal to $5 \Omega$. The corresponding acoustic absorption coefficient is given in Fig. 3, with the label "case 1." It can be observed that the added resistance allows a significative increase of the absorbing capability of the loudspeaker over the bandwidth of interest to the point where it is perfectly absorbent at resonance. This result is at the heart of the principle of shunt loudspeakers, ${ }^{17}$ the stability of which is always ensured with passive dipoles.
Moreover, this electroacoustic solution is an interesting alternative to conventional sound absorbing materials in the low-frequency range, which are often bulky and present poor absorbing efficiency. ${ }^{2}$ But a positive shunt only allows limited values of sound absorption, depending on the total resistances of the loudspeaker system, and no substantial broadening of the bandwidth of control is possible. The intention of Secs. III C and III D is to further extend the preceding properties to active feedbacks, with an emphasis on combined velocity/pressure-feedbacks.

\section{Case 2: Velocity-feedback—shunt negative resistance}

We consider the case where $R_{s}=0 \Omega$ and $\Gamma_{p}=0$ $\mathrm{VPa}^{-1}$, where the only diaphragm velocity feeds back the electroacoustic transducer electrical input. The acoustic absorption coefficient is computed after Eq. (6), the results being reported on Fig. 3, with labels "case 2a" and "case 2b." According to Eq. (13), the EA parameters become:

$$
\left\{\begin{array}{l}
\zeta_{E A, 2}=\frac{1}{Z_{\mathrm{mc}}}\left(R_{\mathrm{ms}}+\frac{B l\left(B l+\Gamma_{v}\right)}{R_{e}}\right) \\
Q_{E A, 2} \approx \frac{1}{R_{e} R_{\mathrm{ms}}+B l\left(B l+\Gamma_{v}\right)} \sqrt{\frac{M_{\mathrm{ms}}}{C_{\mathrm{mc}}}} .
\end{array}\right.
$$

The values of the equivalent mechanical resistance can then be set from a constant value $R_{m s}+(B l)^{2} / R_{e}$, which depends on the loudspeaker's passive resistances and corresponds to the short-circuit case, up to infinity in theory. In the case of high velocity-feedback gains, the control forces the diaphragm to be ideally rigid, corresponding to perfect reflection. Moreover, as feedback gain increases, the quality factor decreases in proportion leading to a consecutive broadening of the control bandwidth.

It is also noticeable that according to Eq. (18), the equivalent shunt of the velocity-feedback is a negative impedance, when $\Gamma_{v}$ takes positive values. Such velocity-feedback, whatever the means to sense velocity, then consists in applying a negative impedance circuit at the transducer electrical terminals, such that:

$$
Z_{2}(s)=-\frac{\Gamma_{v}}{\Gamma_{v}+B l}\left(s L_{e}+R_{e}\right) .
$$

This negative impedance can be obtained with a Wheatston bridge loading the electrical terminals of the loudspeaker, as reported in Ref. 13, confirming the possibility to design EAs capable of self-sensing acoustic quantities out of dedicated electric filters. The stability of velocity-feedback control is theoretically ensured if $\Gamma_{v}$ is positive, according to Eq. (16). In practice though, the setting of the negative impedance is very sensitive to the values of resistance and inductance. An actual limitation of gains is encountered, mainly due to the variation of $L_{e}$ and $R_{e}$ with frequency that are not considered in this model. However, as shown in Sec. IV, there is still a large margin of gains to achieve velocity-feedback without facing instability of the device. 


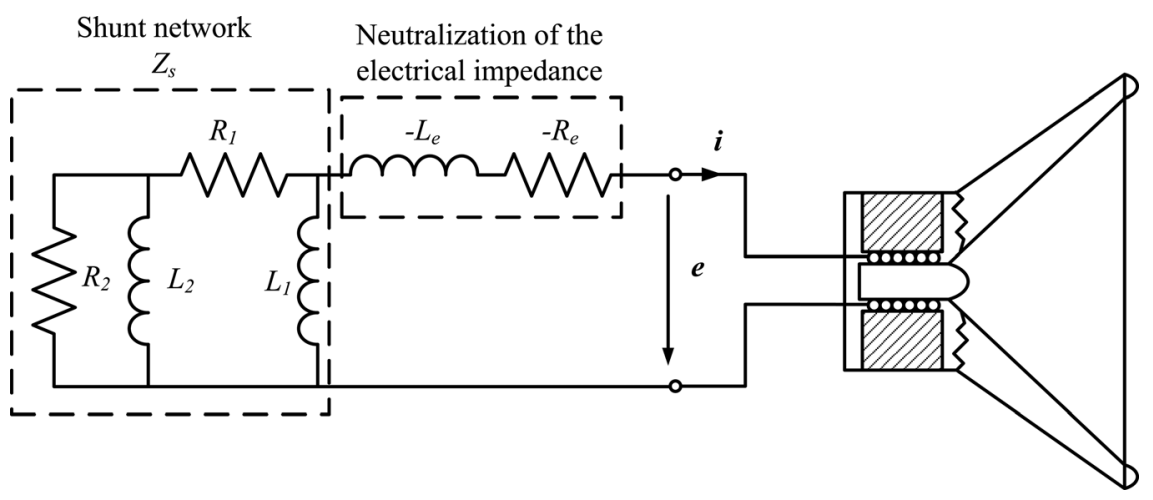

FIG. 4. Example of electric impedance synthesis as an active shunt of an EA.

\section{Case 3: Direct impedance control}

"Direct impedance control" 6 refers to the combination of a velocity- and a pressure-feedback at the loudspeaker electrical terminals. In this case, the normalized acoustic admittance $Y_{3}$ takes the general form of Eq. (8) with $R_{s}=0 \Omega$, and

$$
\left\{\begin{array}{l}
\zeta_{E A, 3} \approx \frac{B l+\Gamma_{v}}{Z_{c} \Gamma_{p}} \approx \frac{1}{Z_{c}} \frac{\Gamma_{v}}{\Gamma_{p}} \\
Q_{E A, 3} \approx \frac{1}{R_{e} R_{\mathrm{ms}}+B l\left(B l+\Gamma_{v}\right)} \sqrt{\frac{M_{\mathrm{ms}}}{C_{\mathrm{mc}}}}
\end{array}\right.
$$

This result indicates that assuming feedback gain $\Gamma_{v} \gg B l$, the target acoustic resistance is directly accessible through the ratio $\Gamma_{v} / \Gamma_{p}$ that should equal in the air at $20{ }^{\circ} \mathrm{C}$ the characteristic impedance is $413.3 \mathrm{~kg} \mathrm{~m}^{-1}$ in view of the total absorption. As for velocity-feedback, the extension of the control bandwidth is made possible by increasing gain $\Gamma_{v}$, theoretically up to infinity, which is hardly the case in practice for the same stability reasons as in the preceding example. The setting of the EA appears quite straightforward, consisting of first adjusting the ratio of gains to equal a desired acoustic resistance value, and then increasing simultaneously the two gains (while their ratio remains constant) up to the above-mentioned instability threshold.

The results given in Fig. 3 with label "case 3a" and "case 3c" demonstrate the possibility of achieving wideband acoustic absorption with such an EA: In these cases, the obtained acoustic impedance matches the target resistance $Z_{c}=\Gamma_{v} / \Gamma_{p}$ on a frequency bandwidth increasing with the value of $\Gamma_{v}$ (decreasing quality factor).

Moreover, the equivalent electric shunt of Eq. (18) is processed with the parameters of case $3 \mathrm{a}$, leading to the function of Eq. (25) illustrated in Fig. 5(a).

$$
Z_{3 a}(s)+\left(s L_{e}+R_{e}\right)=\frac{0.00041 s^{2}+8.5 s}{-0.0019 s^{2}+s-416.44} .
$$

This target electric impedance can be obtained with the electric network illustrated in Fig. 4, composed of electric resistances $\left(R_{1}\right.$ and $\left.R_{2}\right)$ and inductances $\left(L_{1}\right.$ and $\left.L_{2}\right)$. Here, $Z_{s}$ denotes the left part of the electric shunt, excluding the neutralizing electric impedance $-\left(s L_{e}+R_{e}\right)$. In this case,

$$
Z_{s}(s)=\frac{s^{2} L_{2}\left(1+\frac{R_{1}}{R_{2}}\right)+s R_{1}}{s^{2} \frac{L_{2}}{R_{2}}+s\left[1+\frac{L_{2}}{L_{1}}\left(1+\frac{R_{1}}{R_{2}}\right)\right]+\frac{R_{1}}{L_{1}}} .
$$

The identification of the parameters of this network is not straightforward and requires much care, since the number of degrees-of-freedom $\left(R_{1}\right.$ and $R_{2}, L_{1}$ and $\left.L_{2}\right)$ is lower than the number of coefficients of the target electric impedance.
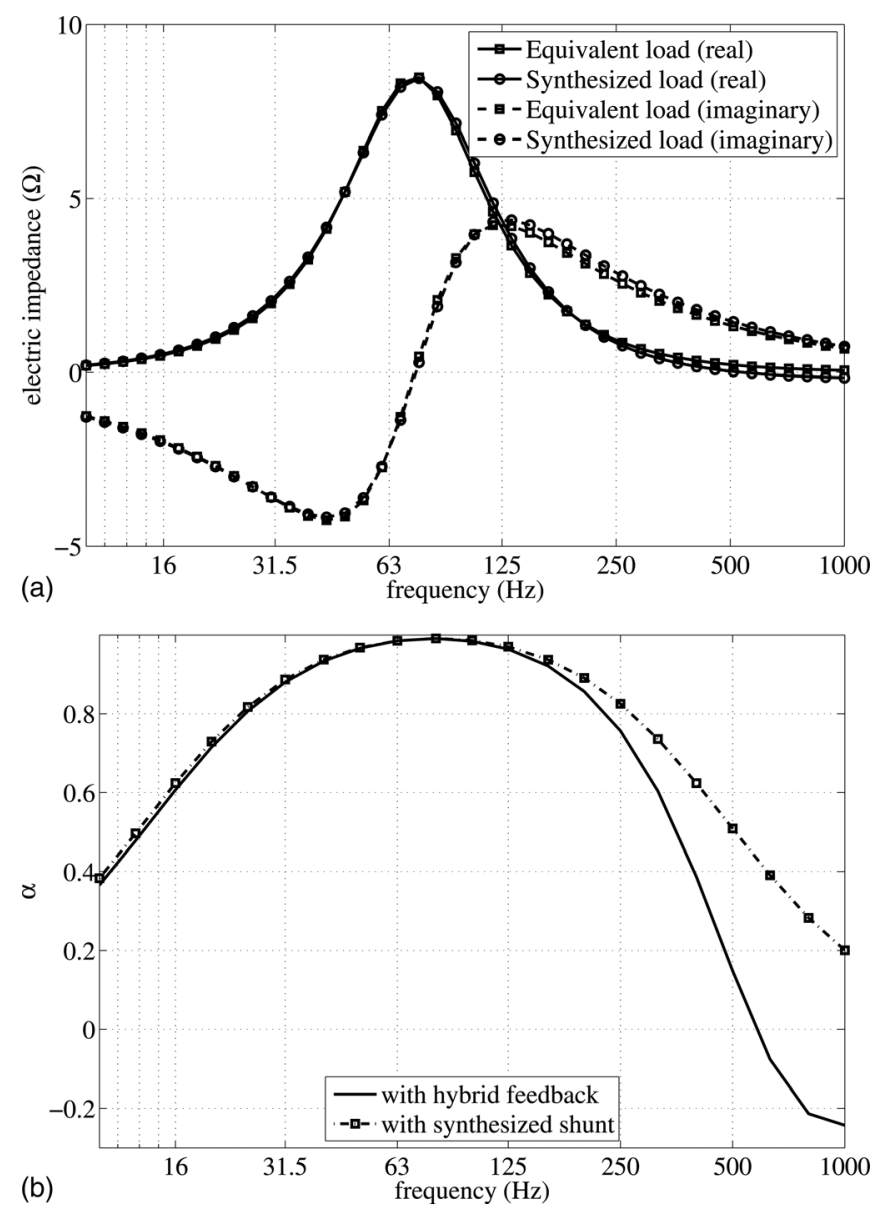

FIG. 5. (a) Simulation of the target electric impedance of Eq. (25) (square markers), and the synthesized electric network impedance of Eq. (28) (round markers) for the "case 3a" (plain lines, real part; dotted lines, imaginary part); (b) Simulation of the acoustic absorption coefficient corresponding to case $3 \mathrm{a}$, with the two equivalent methods (plain line, with direct impedance control; square markers, with the synthesized shunt electric network of Fig. 4). 
Nevertheless, illustrating the equivalence between shunt and feedback control, a set of electric components has been chosen, so that the coefficient of $s^{2}$ on the numerator of Eq. (26) equals 0 , or in other words, the synthesized impedance fits the target one in the low-medium frequency range. It yields,

$$
R_{1}=-R_{2}=8.5 \Omega ; L_{1}=-18.7 \mathrm{mH} ; L_{2}=17.4 \mathrm{mH}
$$

By replacing the values of $\left(R_{1}, R_{2}, L_{1}, L_{2}\right)$ in Eq. (26), one can obtain the following synthesized impedance:

$$
Z_{s}(s)=\frac{8.5 s}{-0.0020 s^{2}+s-454.5},
$$

that can be compared to the expression of Eq. (25), as illustrated in Fig. 5(a). Thus, the synthesized electric impedance matches the target one within the frequency bandwidth of interest.

Conversely, this synthesized electric impedance $Z_{s}(s)$ forms a new shunt impedance in series with $-\left(s L_{e}+R_{e}\right)$ that we can then substitute for $R_{s}$ in Eq. (7) [or even Eq. (20)] to compute the corresponding "synthesized" acoustic admittance denoted by $Y_{s}, 3 a$ (in order to distinguish this synthesized admittance and the target one $Y_{3 a}$ ). The synthesized acoustic admittance is then:

$$
\begin{aligned}
& Y_{s, 3 a}(s)= \\
& Z_{\mathrm{mc}} \frac{s}{s^{2}\left(M_{\mathrm{ms}}+(B l)^{2} \frac{L_{2}}{R_{1} R_{2}}\right)+s\left(R_{\mathrm{ms}}+\frac{(B l)^{2}}{R_{1}}\right)+\left(\frac{1}{C_{\mathrm{mc}}}+\frac{(B l)^{2}}{L_{1}}\right)}
\end{aligned}
$$

This normalized admittance, with the chosen values of Eq. (27), corresponds to a theoretically stable configuration of the EA, according to the Routh criterion, assuming that the neutralization of the electric impedance is ideally achieved. The "synthesized" absorption coefficient is then processed, according to Eqs. (6) and (29), and compared in Fig. 5(b) to the one obtained with direct impedance control (with $\Gamma_{v}=10 \mathrm{~V} \mathrm{~m}^{-1} \mathrm{~s}, \Gamma_{p}=0.025 \mathrm{~V} \mathrm{~Pa}^{-1}, R_{s}=0 \Omega$ ).

This last result illustrates the formal equivalence between shunt loudspeakers and feedback-based active sound absorption showing similar results in terms of sound absorption. One can observe that, with the chosen electric network, the coefficients of $s^{2}$ and $s^{0}$ in the denominator of the synthesized acoustic admittance $Y_{s, 3 a}(s)$ are lower than in the passive case [see Eq. (19)]. This is in accordance with the objective of lowering the equivalent mass and increasing the equivalent compliance of the loudspeaker in order to extend the bandwidth of the control. Moreover, the equivalent acoustic resistance is actually higher than the passive one, which is required to match the acoustic resistance of air. The electrical network allows the adjustment of the three parameters of the acoustic resonator to the target. This result opens the way to new strategies for the optimization of electric networks shunting a loudspeaker in view of active sound absorption. Practically, such electric impedance design is not straightforward and needs a very accurate selection of the electric components, especially with respect to stability, but also in terms of absorption performances. The implementa- tion of such impedance synthesis strategy on digital signal processing platforms could help alleviate these issues, but these developments are out of the scope of this paper.

\section{E. Discussions}

The results in Fig. 3 (see Table II for control parameters) clearly highlight the similarities between the different control techniques detailed in Secs. III A-D, unifying passive shunt techniques and active feedback control of acoustic impedance into a single formalism. The passive performances of an EA can be first improved with a simple passive electric resistance of optimal value so as to reach almost perfect absorption within a narrow frequency bandwidth around the resonance due to the increase of total resistances and the slight decrease of the resonance quality factor. The bandwidth of control can then be significantly increased by choosing appropriate feedback gains in a combined pressure-velocity-feedback. This leads to an enhanced damping at resonance, and a highly decreased quality factor of resonance, resulting from the lowering of the apparent mass and compliance of the resonator. Inspired by these formal analogies, a technique for adjusting the active feedback gains is also introduced. It consists in tuning the three independent parameters of the equivalent acoustic resonator, presenting interesting perspectives for controlling the acoustic impedance of an electroacoustic loudspeaker. As long as the considered lumped-element model is valid [at least up to the cut-off frequencies of Eq. (10)], a criterion for stability has been identified allowing a wide range of settings for the resonator parameters. On the other hand, the acoustic performances obtained with synthesized electric impedances theoretically match the ones obtained with feedbacks on acoustic quantities. The synthesis of electric impedances is also shown to be theoretically possible with quite simple electric networks, such as the one of Fig. 4, assuming that the neutralization of the loudspeaker electric impedance has been primarily performed with accuracy. But, in practice, this is very sensitive to the electric components, and stable analog implementations have been difficult to be realized at this stage. However, this theoretical result still presents interesting properties of EAs and bridges a conceptual gap between shunt loudspeakers and feedback-based active acoustic impedance control. Section IV intends to experimentally validate the aforementioned properties.

\section{EXPERIMENTAL ASSESSMENT}

In order to assess experimentally the equivalence between the active feedback control and electric shunts, a closed-box (volume $V_{b}=10$ l) Visaton ${ }^{\circledR}$ AL 170 low-midrange loudspeaker is employed as an EA. The acoustic absorption coefficient of the EA is assessed after ISO 10534-2 standard, ${ }^{26}$ as described in Fig. 6. In this setup, an impedance tube is specifically designed (length $\mathrm{L}=3.4 \mathrm{~m}$; internal diameter $\varnothing=150 \mathrm{~mm}$ ), one termination of which is closed by an EA, the other extremity being open with a horn-shape termination so as to exhibit anechoic conditions. ${ }^{27}$ A source loudspeaker is wall-mounted close to this termination. Two holes located at positions $x_{1}=0.46 \mathrm{~m}$ and $x_{2}=0.35 \mathrm{~m}$ from 


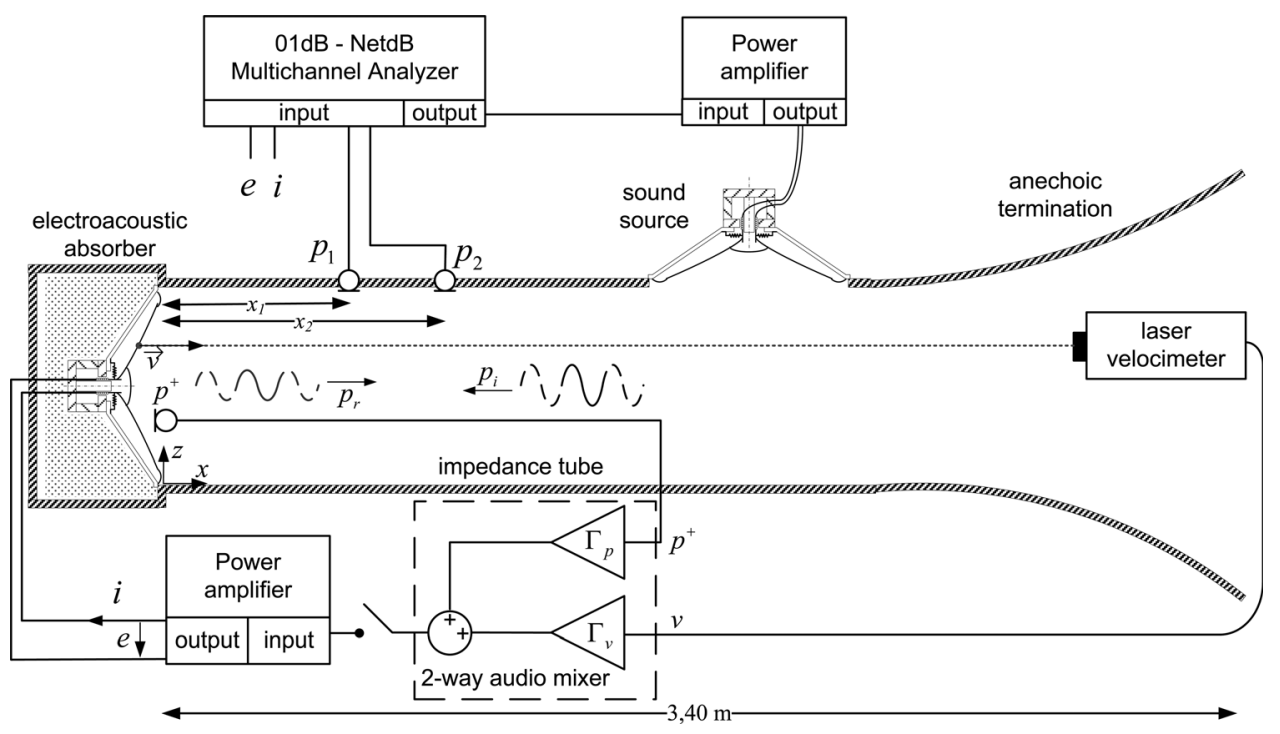

FIG. 6. Experimental setup for the assessment of EAs absorption Coefficient.

the EA position are the receptacles of $1 / 2^{\prime \prime}$ microphones (Norsonic Type 1225 cartridges mounted on Norsonic Type 1201 amplifiers), sensing sound pressures $p_{1}=p\left(x_{1}\right)$ and $p_{2}=p\left(x_{2}\right)$ and the transfer function $H_{12}=p_{2} /\left(p_{1}\right)$ is processed through a 01dB-NetdB Multichannel Analyzer. Simultaneously, with a view to process the equivalent electric load $Z$ at the EA electric terminals, the electric voltage $e$ and current $i$ circulating through the coil are measured and processed with the same instrumentation.

In this experimental study, the active feedback settings corresponding to case $2 \mathrm{c}$ and case $3 \mathrm{~b}$ have been applied at the EA electric terminals, as well as at the optimal shunt of case 1 . Here, the velocity-feedback is processed through a Polytec OFV-505/5000 laser velocimeter (sensitivity being set to $\sigma_{v}=100 \mathrm{~V} \mathrm{~m}^{-1} \mathrm{~s}$ ). This velocity sensor is positioned at the output of the open tube, as illustrated in Fig. 6-the laser beam focusing on a single point of the radiator at the middle of its radius. The pressure is sensed with an external PCB 130D20 microphone (sensitivity of $\sigma_{p}=47.5 \mathrm{mV} \mathrm{Pa}^{-1}$ ) located in the plane $x=0$ and slightly off-center (at a height of $z=3.2 \mathrm{~cm}$ from the duct wall), yielding a distance of approximately $5 \mathrm{~mm}$ from the loudspeaker diaphragm. The direct impedance control is processed through a two-way analog audio-mixer, allowing the setting of electric feedback gains $\Gamma_{v}^{\prime}=\Gamma_{v} /\left(\sigma_{v}\right)$ and $\Gamma_{p}^{\prime}=\Gamma_{p} /\left(\sigma_{p}\right)$. The sound absorption coefficients measured with the above-mentioned setup are compared to the corresponding model simulations described in Sec. III and illustrated in Fig. 7(a). In parallel, the equivalent electric load, processed as the transfer function between voltage $e$ and current $i$ at the EA terminals, is assessed for the settings of case $3 \mathrm{~b}$ and compared to the corresponding model simulation given in Fig. 7(b).

The absorption coefficient presented at the front face of the loudspeaker can be easily varied from almost total reflection up to total absorption over a wide frequency bandwidth depending on the control case. The theoretical curves show the same trend as the experimental values even if some slight differences can be observed with the ISO 10534-2 technique. These measurements also show a similar behavior of different acoustic resonators, presenting variable acoustic resistan- ces and quality factors, given in Table II. With such formalism, wide-band acoustic absorbers can be designed in a very straightforward manner according to certain specifications: If an application requires narrow-band absorption,
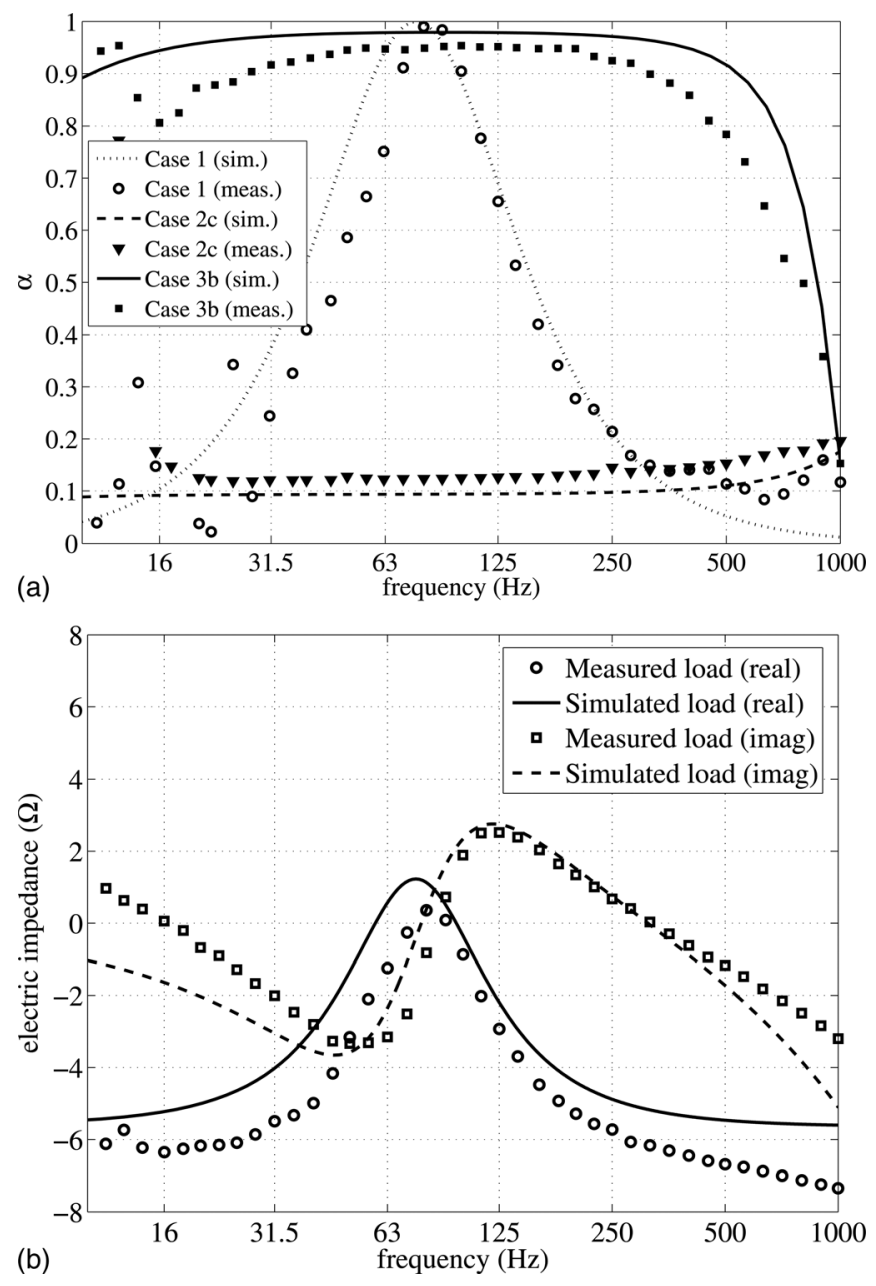

FIG. 7. Experimental assessment of the EA and comparison to numerical simulations: (a) absorption coefficient obtained with optimal shunt (case 1: $R_{s}=5 \Omega$ ) velocity-feedback (case $2 \mathrm{c}: \Gamma_{v}=194 \mathrm{~V} \mathrm{~m}^{-1} \mathrm{~s}$ ) and direct impedance control (case 3b: $\Gamma_{\mathrm{v}}=70.0 \mathrm{~V} \mathrm{~m}^{-1} \mathrm{~s}$ and $\Gamma_{\mathrm{p}}=0.13 \mathrm{~V} \mathrm{~Pa}^{-1}$ ); (b) measured and simulated equivalent electric load for case $3 \mathrm{~b}$. 

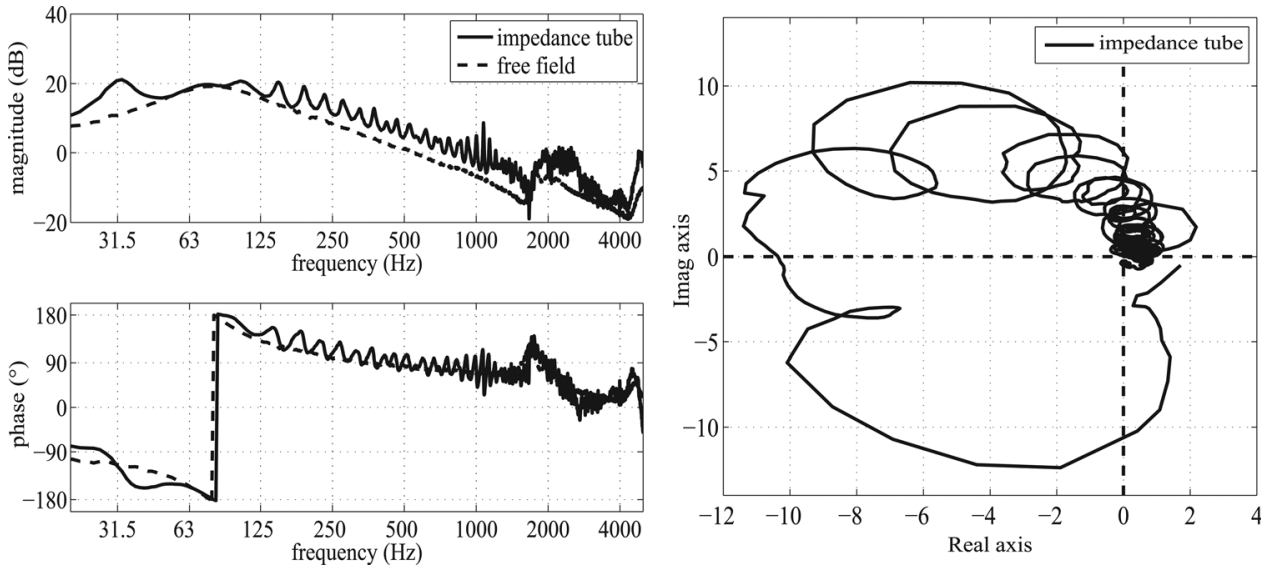

FIG. 8. Experimental assessment of the open-loop gain (left upper chart, magnitude in decibel; left lower chart, phase in degrees; right chart, Nyquist plot) for the direct impedance control setting (case $3 b$ ) in different acoustic environments (plain lines, in the impedance tube; dotted lines, in the anechoic chamber).

passive shunt can easily be deployed, and in other cases stable and still simple active impedance control can readily be set. Moreover, the electric assessment confirms that, when connected to an active feedback control device, the EA behaves as if it was connected to an active electric load, the elements of which can be identified on the measured transfer function. These assessed values also match the transfer function of the electric network given as example in Sec. III D. Slight discrepancies are observed between the theory and experimental data, confirming the importance of accurately identifying the components of the loudspeaker to be employed in the active device. For example, it appears in Fig. 7(b) that the dependence of $R_{e}$ and $L_{e}$ with frequency should be accurately modeled with a view to synthesizing the requested electric network.

On the stability side, all the active control gains presented in this paper have been set under the threshold of instability. In practice, instability can be experienced while further increasing the feedback gains, the threshold depending on the reactive components in the electroacoustic loudspeaker (especially the electric inductance), as explained in Sec. II B 2. As an illustration, the loop stability is assessed for the settings of case $3 \mathrm{~b}$, according to the stability criterion presented in Ref. 28 for single channel feedback control. This argument says that, if the phase of the transfer function between the input and the output at the disconnection in the loop is $360^{\circ}$, then the magnitude of the transfer function should be less than unity. Here, the open-loop gain is measured with the $01 \mathrm{~dB}-$ NetdB Multichannel Analyzer processing the transfer function between the signals provided at the input of the power amplifier feeding the EA and the output of the audio-mixer. This open-loop gain is first measured when the EA is placed at the entrance of the impedance tube in Fig. 7, and in a second step in the case where it is moved to an anechoic chamber (free-field conditions). The comparison is illustrated in Fig. 8. The results are presented on an extended bandwidth $(10-5000 \mathrm{~Hz})$ to identify potential highfrequency effects.

Generally speaking, the main problem might arise at the resonance frequency of the EA, where the open-loop gain shows the highest magnitude. This problem is easily alleviated by choosing appropriate settings for the two feedback gains. Indeed, for a certain target acoustic impedance $\Gamma_{v} /\left(\Gamma_{p}\right)$, there always exists a combination of the two feed- back gains $\Gamma_{v}$ and $\Gamma_{p}$ yielding a $180^{\circ}$ phase rotation at this frequency. Apart from this problem, stability issues could also arise from the reactive component of the EA, such as the electric inductance of the coil, or higher-order resonances of the diaphragm, as can be observed above $1 \mathrm{kHz}$ on the free-field measurement. These problems might generally represent limitations for setting the feedback gains, but do not actually affect the acoustic performances of the device in the frequency bandwidth of interest.

However, the main instability issues occurring during the assessments appear to be related to the experimental facility. In the impedance tube especially, the open-loop gain illustrated in Fig. 8 presents a gain margin of about $2 \mathrm{~dB}$ (at $2641 \mathrm{~Hz}$ ) and a phase margin of $2.7^{\circ}$ (at $4781 \mathrm{~Hz}$ ). One can observe that these margins are significantly increased in the case where the acoustic absorber is in a free-field environment; the resonances of the impedance tube represent the most important factor of magnitude and phase variations in the open-loop gain. As a conclusion, the observed instability issues should not be entirely taken for an intrinsic property of the EA, and the margins for setting the EAs are actually much higher than the ones assessed in the impedance tube.

\section{CONCLUSIONS}

A unifying theory of active acoustic impedance control has been introduced, covering different control techniques from passive shunt to pressure/velocity-feedbacks in a single formalism. A feedback on acoustic quantities is shown to be equivalent to an electrical load at the transducer electrical terminals. Conversely, a synthetic electric network has been identified for each active acoustic impedance control, the design of which can be specified in a relatively simple manner. Broadband acoustic performances have been measured on a generic prototype of EA with passive shunt and active acoustic feedback control. The tested configurations present a variety of acoustic absorption, which are in good agreement with the simulations. Finally, the equivalent electric load of an active acoustic feedback has also been experimentally assessed, confirming the theory of electroacoustic absorbers.

Further work is ongoing, focusing on the design and optimization of dedicated electrical networks. The optimization addresses the identification of the target electric 
impedance expressed in Eq. (26), along the same methodology as the one reported for optimizing semi-active shunt loudspeakers. ${ }^{29}$ A potential application of the reported concepts could consist in designing specified electric filters capable of sensing acoustic quantities (pressure and/or diaphragm velocity) out of electrical current/voltage, thus preventing the use of external sensors in active noise control devices.

\section{ACKNOWLEDGMENTS}

This work was supported by the Swiss National Science Foundation under Research Grant No. 200021-116977. The authors also wish to thank Patrick Roe and Travis Forbes for their helpful reviewing of the manuscript.

${ }^{1}$ P. Lueg, "Process of silencing sound oscillations," U.S. patent no US2043416 (1936).

${ }^{2}$ H. F. Olson and E. G. May, "Electronic sound absorber," J. Acoust. Soc. Am. 25, 1130-1136 (1953).

${ }^{3}$ M. J. M. Jessel and G. Mangiante, "Active sound absorbers in an air duct," J. Sound Vib. 23, 383-390 (1972).

${ }^{4}$ D. Guicking and E. Lorenz, "An active sound absorber with porous plate," ASME J. Vib. Acoust. Stress Reliab. Des. 106, 389-392 (1984).

${ }^{5}$ C. Guigou and C. R. Fuller, "Adaptive feedforward and feedback methods for active/passive sound radiation control using smart foam," J. Acoust. Soc. Am. 104, 226-231 (1998).

${ }^{6}$ M. Furstoss, D. Thenail, and M. A. Galland, "Surface impedance control for sound absorption: Direct and hybrid passive/active strategies," J. Sound Vib. 203, 219-236 (1997).

${ }^{7}$ D. Guicking, K. Karcher, and M. Rollwage, "Coherent active methods for applications in rooms acoustics," J. Acoust. Soc. Am. 78, 1426-1434 (1985).

${ }^{8}$ O. Bustamante and P. Nelson, "An adaptive controller for the active absorption of sound," J. Acoust. Soc. Am. 91, 2740-2747 (1992).

${ }^{9} \mathrm{P}$. Darlington, "Loudspeaker circuit with means for monitoring the pressure at the speaker diaphragm, means for monitoring the velocity of the speaker diaphragm and a feedback circuit," World patent no. WO9703536 (1997).

${ }^{10}$ M. Collet, P. David, and M. Berthillier, "Active acoustical impedance using distributed electrodynamical transducers," J. Acoust. Soc. Am. 125, 882-894 (2009).
${ }^{11}$ C. H. Hansen, Understanding Active Noise Cancellation (Spon Press, London, 2001), pp. 69-110.

${ }^{12}$ R. E. Werner, "Loudspeakers and negative impedances,", IRE Trans. Audio 6, 83-89 (1958).

${ }^{13}$ E. De Boer, "Theory of motional feedback," IRE Trans. Audio 9, 15-21 (1961).

${ }^{14}$ S. A. Lane and R. L. Clark, "Improving loudspeaker performance for active noise control applications,” J. Audio Eng. Soc. 46, 508-519 (1998).

${ }^{15}$ R. J. Bobber, "An active transducer as a characteristic impedance of an acoustic trans mission line," J. Acoust. Soc. Am. 48, 317-324 (1970).

${ }^{16}$ S. J. Elliott, P. Joseph, P. A. Nelson, and M. E. Johnson, "Power output minimization and power absorption in the active control of sound," $\mathrm{J}$. Acoust. Soc. Am. 90, 2501-2512 (1991).

${ }^{17}$ A. J. Fleming, D. Niederberger, S. O. R. Moheimani, and M. Morari, "Control of resonant acoustic sound fields by electrical shunting of a loudspeaker,” IEEE Trans. Control Syst. Technol. 15, 689-703 (2007).

${ }^{18}$ H. Lissek, "Les materiaux actifs a proprietes acoustiques variables (Active materials with variable acoustic properties)," Ph.d. dissertation, Universite du Maine (2002).

${ }^{19} \mathrm{H}$. Lissek and X. Meynial, "A preliminary study of an isodynamic transducer for use in active acoustic materials," Appl. Acoust. 64, 917-930 (2003).

${ }^{20}$ X. Meynial, "Active Acoustic Impedance Control Device," World patent no. WO9959377 (1999).

${ }^{21}$ T. Sluka, P. Mokry, and H. Lissek, "A theory of sound transmission through a clamped curved piezoelectric membrane connected to a negative capacitor,” Int. J. Solids Struct. 47, 2260-2267 (2010).

${ }^{22}$ M. Rossi, Audio (Presses Polytechniques et Universitaires Romandes, Lau sane, 2007), pp. 533-555.

${ }^{23}$ W. S. Levine, The Control Handbook (Chemical Rubber Company Press, Boca Raton, FL, 1996), pp. 131-135.

${ }^{24}$ W. Klippel, “Tutorial: Loudspeaker nonlinearities. Causes, parameters, symptoms," J. Audio Eng. Soc 54, 907-939 (2006).

${ }^{25}$ R. Ravaud, G. Lemarquand, and T. Roussel, “Time-varying non linear modeling of electrodynamic loudspeakers," Appl. Acoust. 70, 450-458 (2009).

${ }^{26}$ ISO 10534-2-1998: Acoustics Determination of Sound Absorption Coefficient and Impedance in Impedance Tubes. Part 2: Transfer-function Method (ISO, Geneva, Switzerland, 1998).

${ }^{27}$ ISO 5136-2003: Acoustics-Determination of Sound Power Radiated into a Duct by Fans and Other Air-moving Devices-In-duct Method (ISO, Geneva, Switzerland, 2003).

${ }^{28} \mathrm{P}$. Nelson and S. Elliott, Active Control of Sound (Academic Press, London, 1993), pp. 211-214.

${ }^{29}$ R., Boulandet and H. Lissek, "Optimization of electroacoustic absorbers by means of designed experiments," Appl. Acoust. 71, 830-842 (2010). 